\title{
L'avenir de la chirurgie pédiatrique en France : la démographie des chirurgiens pédiatres en formation
}

\section{The future of pediatric surgery in France: demographics of pediatric surgery residents and fellows}

\author{
Aurélien Scalabre ${ }^{1,2}$, Emeline Bourgeois ${ }^{1,3}$, Matthieu Peycelon ${ }^{1,4}$, \\ Maguelonne PoNs ${ }^{1,5}$, Sébastien RAUX ${ }^{6}$, Rémi KOHLER ${ }^{7,8}$ et François VARLET ${ }^{7,9}$ \\ 1 Association des chirurgiens pédiatres en formation \\ 2 Service de chirurgie pédiatrique uro-génitale, viscérale et thoracique, Hôpital Femme Mère Enfant, \\ 59 boulevard Pinel, 69500 Bron, France \\ 3 Service orthopédie traumatologie. Hôpital nord Centre hospitier universitaire (CHU), Grenoble, BP 217, \\ 38700 La Tronche, France \\ 4 Service de Chirurgie Infantile Viscérale, Hôpital Robert Debré, 48 boulevard Sérurier, 75935 Paris Cedex 19, France \\ 5 Service de chirurgie pédiatrique, American Memorial Hospital, 47 rue Cognacq-Jay, 51092 Reims Cedex, France \\ 6 Département de chirurgie infantile et de traumatologie, Hôpital Jeanne de Flandre, Avenue Eugène Avinée, \\ 59037 Lille Cedex, France \\ 7 Collège national de chirurgie pédiatrique, France \\ 8 Service de chirurgie pédiatrique orthopédique et traumatologique, Hôpital Femme Mère Enfant, 59 boulevard Pinel, \\ 69500 Bron, France \\ 9 Service de chirurgie pédiatrique, CHU Hôpital Nord, 42055 St-Étienne, France
}

Manuscrit reçu le 12 juillet 2010; commentaires éditoriaux formulés aux auteurs le 12 février 2011; accepté pour publication le 20 février 2011

\author{
Mots clés : \\ Démographie ; \\ internat ; \\ chirurgie pédiatrique ; \\ formation ; \\ diplôme d'études \\ spécialisées \\ complémentaires
}

Résumé - Objectifs : Faire le recensement des internes et chefs de clinique des universitésassistants des hôpitaux (CCA) en formation en chirurgie pédiatrique et analyser les résultats en fonction des besoins de la spécialité. Matériel et méthodes : Les données concernant la démographie des chirurgiens pédiatres en formation ont été recueillies par un questionnaire adressé aux internes, CCA et coordonnateurs interrégionaux de la spécialité, de septembre 2009 à mars 2010. Les informations suivantes ont été recueillies : nom et prénom, sexe, année de validation du concours de l'internat ou des épreuves classantes nationales, orientation choisie en chirurgie pédiatrique, le centre hospitalier universitaire (CHU) de formation durant l'internat, lieu d'exercice. Résultats : Cinquante-trois internes sont inscrits en diplôme d'études spécialisées complémentaires (DESC) de chirurgie infantile avec une orientation urologique, viscérale, thoracique ou plastique et vingt-et-un en orthopédie pédiatrique. Par ailleurs treize internes en DESC d'orthopédie adulte vont s'orienter vers l'orthopédie pédiatrique. La répartition des internes sur le territoire montre des différences importantes entre les inter-régions. Il existe 55 postes de CCA en chirurgie pédiatrique. Conclusion : Cette étude devra servir de socle aux réflexions engagées par la Commission nationale de l'internat 
et du post-internat. Le nombre important d'internes formés chaque année montre l'attractivité de la chirurgie pédiatrique pour les chirurgiens en formation. Il nous alerte sur la nécessité d'adapter la formation des chirurgiens pédiatres aux capacités de formation de notre système de santé et aux besoins de santé de la population française.

Keywords: Demography; internship; pediatric surgery; surgical training
Abstract - Objective: To record the number of pediatric surgery residents and fellows in France. Material and methods: Demographic data about pediatric surgery residents and fellows was collected through a questionnaire sent to every resident, fellow and regional coordinator between September 2009 and March 2010. Results: Fifty-three residents are enrolled in the French pediatric surgery training program and 21 in the French orthopedic pediatric surgery program. Thirteen residents in the adult orthopedic surgery training program are specializing in pediatric orthopedic surgery. The geographic distribution highlights striking differences between regions. Fifty-five surgical fellowship positions in pediatric surgery are currently available. Conclusion: This study should help the French national internship and post-internship commission better plan the future of French pediatric surgery. The large number of residents trained each year is proof that pediatric surgery can draw surgery residents. It demonstrates the need to adjust the training program to the health system's capabilities and the needs of the French population.

\section{Introduction}

Dans le contexte actuel de réforme du système de santé français, la démographie et la formation des chirurgiens sont des enjeux majeurs.

La Commission nationale de l'internat et du post-internat (CNIPI) a été créée par décret le 9 mars 2009 et joue un rôle consultatif concernant la répartition et la formation des internes ${ }^{[1]}$. Elle doit consulter les représentants de chaque spécialité afin de définir les effectifs d'internes à former par spécialité et par région, le déroulement de la maquette de formation ainsi que les conditions d'accès aux diplômes d'études spécialisées complémentaires (DESC). Elle mène une réflexion constructive sur les problématiques liées au post-internat.

La chirurgie pédiatrique est une spécialité passionnante pour les chirurgiens en formation et les internes sont plus nombreux chaque année à choisir cette carrière.

Dans le cadre de cette commission, il était donc nécessaire de réaliser une étude de la démographie des futurs chirurgiens pédiatres.

Le Collège national hospitalier et universitaire de chirurgie pédiatrique (CNHUCP) et l'Association des chirurgiens pédiatres en formation (ACPF) ont réalisé cette étude dont les objectifs étaient de faire le recensement des internes et chefs de clinique des universités-assistants des hôpitaux (CCA) en chirurgie pédiatrique et d'analyser les résultats en fonction des besoins de formation de la spécialité et des besoins de santé de la population.

\section{Contexte académique de la formation des chirurgiens pédiatres en France}

En France, l'orientation des étudiants en médecine vers les différentes filières spécialisées résulte, depuis 1984, d'une procédure sélective fondée sur un classement national des étudiants, organisé en fin de second cycle (internat puis épreuves classantes nationales). Les étudiants en médecine choisissent la région où ils effectueront leur internat qualifiant, ainsi que leur diplôme d'études spécialisées (DES). L'organisation de l'enseignement du DES est inter-régionale. L'obtention du DES au terme de l'internat qualifiant (d'une durée de dix semestres pour les spécialités chirurgicales) est en principe suffisante pour la délivrance d'autorisation d'exercice ordinale ; cependant, pour certaines spécialités, il est nécessaire d'obtenir également un DESC. Ainsi, pour exercer la chirurgie pédiatrique, 
il convient d'obtenir le DES de chirurgie générale puis le DESC de chirurgie infantile.

Le CNHUCP organise l'enseignement du DESC de chirurgie infantile, qui a lieu sous forme de cours semestriels portant sur l'ensemble du programme de la spécialité, organisé en cycles de trois ans. L'ACPF a pour vocation de représenter les internes de chirurgie pédiatrique auprès des sociétés savantes et contribue à l'organisation des cours de DESC en collaboration avec le CNHUCP.

L'obtention du DESC de chirurgie infantile nécessite la validation d'une épreuve écrite organisée au niveau national en cinquième et dernière année d'internat, suivie d'une épreuve orale régionale durant la première année de post-internat.

L'obtention du DESC exige donc de valider un post-internat d'une durée de deux à quatre ans, en assurant les fonctions d'assistant spécialiste, d'assistant hospitalo-universitaire (AHU) ou de CCA. Le post-internat permet aux jeunes médecins d'approfondir leur formation spécialisée sur des fonctions de plein exercice, avec également un rôle universitaire d'enseignement et de recherche pour les CCA et les AHU. La création de tels postes ne dépend pas des seuls centres hospitaliers universitaire (CHU) ni des seules facultés mais résulte d'une décision du ministère de la santé pour les postes d'assistants spécialistes et du ministère de l'enseignement supérieur pour les postes de CCA. Dans ce contexte, il est nécessaire de disposer de données démographiques précises et évolutives, afin d'articuler au mieux les besoins médico-économiques et les besoins de formation, à plus forte raison pour les spécialités dont les effectifs sont réduits, comme c'est le cas en chirurgie pédiatrique.

\section{Matériel et méthodes}

Les données démographiques concernant les internes en formation en chirurgie pédiatrique ont été recueillies de septembre 2009 à mars 2010. Les internes et CCA ont été invités à remplir un questionnaire et le même questionnaire a été envoyé par courrier électronique à ceux ayant assisté au moins une fois aux cours de DESC pour une meilleure exhaustivité.

Ce questionnaire a permis de recueillir les informations suivantes : nom, prénom, sexe, année de validation du concours de l'internat ou des épreuves classantes nationales (ECN), orientation choisie en chirurgie pédiatrique urologique, viscérale, thoracique et plastique ou orthopédique (le DESC de chirurgie infantile est unique mais constitué deux sous-groupes selon la sur-spécialité choisie par le candidat; l'enseignement est organisé en trois parties : tronc commun, uro-viscéral et orthopédie pédiatrique), centre hospitalier universitaire (CHU) de formation durant l'internat, lieu d'exercice pour les CCA, orientation envisagée pour les cinq ans à venir.

Les enseignants coordonnateurs inter-régionaux de chirurgie pédiatrique réunis lors du conseil d'administration du CNHUCP du 29 janvier 2010 ont permis de préciser les effectifs d'internes et de CCA inscrits en DESC dans chaque inter-région, ainsi que les postes de CCA existants dans chaque centre hospitalier et leur orientation en chirurgie pédiatrique urologique, viscérale, thoracique et plastique ou orthopédique.

La totalité des internes et CCA sollicités ont répondu au questionnaire. Les coordonnateurs interrégionaux ont fourni l'ensemble des informations demandées sur les internes et CCA inscrits en DESC. Les internes et CCA pour lesquels certaines informations manquaient à l'issue de ces démarches ont été contactés par courrier électronique ou par téléphone. Ce recueil d'informations a permis de préciser la démographie actuelle des chirurgiens pédiatres en formation.

\section{Résultats}

\section{Effectifs des internes}

Soixante-quatorze internes sont inscrits en DESC de chirurgie infantile, cinquante-trois en chirurgie pédiatrique viscérale et thoracique et vingt-et-un en orthopédie pédiatrique. Les effectifs sont résumés dans le tableau I par année de validation de l'internat ou de l'ECN et dans le tableau II par inter-région ; $66 \%$ 
Tableau I. Effectifs des internes de chirurgie pédiatrique inscrits en diplôme d'études spécialisées complémentaires (DESC) par année de validation de l'internat ou des épreuves classantes nationales (ECN).

\begin{tabular}{|c|c|c|c|}
\hline $\begin{array}{c}\text { Année de } \\
\text { validation de } \\
\text { l'internat } \\
\text { (ou ECN) }\end{array}$ & $\begin{array}{c}\text { Chirurgie } \\
\text { pédiatrique } \\
\text { viscérale, } \\
\text { urologique, } \\
\text { thoracique } \\
\text { et plastique }\end{array}$ & $\begin{array}{c}\text { Orthopédie } \\
\text { pédiatrique }\end{array}$ & $\begin{array}{c}\text { Orthopédie } \\
\text { adulte } \\
\text { s'orientant } \\
\text { vers } \\
\text { l'orthopédie } \\
\text { pédiatrique }\end{array}$ \\
\hline 2004 & 7 & 0 & 0 \\
\hline 2005 & 18 & 6 & 6 \\
\hline 2006 & 17 & 3 & 4 \\
\hline 2007 & 9 & 11 & 2 \\
\hline 2008 & 1 & 1 & 1 \\
\hline Total & 53 & 21 & 13 \\
\hline
\end{tabular}

des internes inscrits en DESC de chirurgie pédiatrique sont des femmes et $34 \%$ sont des hommes. Treize internes inscrits en DESC d'orthopédie adulte ont dit vouloir s'orienter ensuite vers l'orthopédie pédiatrique. Parmi les promotions 2009 et 2010, 21 internes supplémentaires souhaiteraient s'inscrire en DESC de chirurgie infantile.

\section{Répartition sur le territoire}

L'inter-région Ouest, comprenant aussi les départements et territoires d'Outre-Mer (DOM-TOM), est celle dans laquelle sont formés le plus grand nombre d'internes de chirurgie pédiatrique à orientation viscérale avec quatorze inscrits. À l'inverse quatre internes «seulement » sont inscrits dans ce DESC en Île-de-France.

Pour l'orthopédie pédiatrique, l'inter-région dans laquelle le plus grand nombre d'internes est formé est l'inter-région Nord avec sept internes inscrits en DESC de chirurgie infantile avec option orthopédie et aucun interne issu du DESC d'orthopédie adulte. À l'inverse, en Île-de-France, aucun interne n'est inscrit en DESC de chirurgie infantile avec option orthopédie mais huit internes sont inscrits en DESC d'orthopédie adulte avec une orientation probable vers l'orthopédie pédiatrique.
Tableau II. Effectifs des internes de chirurgie pédiatrique inscrits en diplôme d'études spécialisées complémentaires (DESC) par inter-région.

\begin{tabular}{|c|c|c|c|}
\hline $\begin{array}{c}\text { Internes } \\
\text { inscrits } \\
\text { en DESC } \\
\text { par } \\
\text { inter-région }\end{array}$ & $\begin{array}{c}\text { Chirurgie } \\
\text { pédiatrique } \\
\text { viscérale, } \\
\text { urologique, } \\
\text { thoracique } \\
\text { et plastique }\end{array}$ & $\begin{array}{c}\text { Orthopédiatrique } \\
\text { pédithopédie } \\
\text { adulte } \\
\text { s'orientant } \\
\text { vers } \\
\text { l'orthopédie } \\
\text { pédiatrique }\end{array}$ \\
\hline Nord & 8 & 7 & 0 \\
\hline $\begin{array}{c}\text { Ouest et } \\
\text { Départements } \\
\text { d'Outre Mer }\end{array}$ & 14 & 3 & 1 \\
\hline Sud-Ouest & 8 & 3 & 0 \\
\hline Sud-Est & 5 & 1 & 2 \\
\hline Rhône Alpes & 5 & 2 & 1 \\
\hline $\begin{array}{c}\text { Paris } \\
\text { Île-de-France }\end{array}$ & 4 & 0 & 8 \\
\hline Est & 9 & 5 & 1 \\
\hline Sous-total & & 21 & 13 \\
\hline Total & 53 & & 34 \\
\hline
\end{tabular}

\section{Le post-internat}

Le nombre de postes de CCA ou d'AHU existant au $1^{\mathrm{er}}$ mai 2010 en chirurgie pédiatrique a été déterminé par le questionnaire envoyé aux coordonnateurs interrégionaux. Les effectifs sont résumés dans le tableau III. Il existe vingt-huit postes en chirurgie pédiatrique à orientation urologique, viscérale, thoracique ou plastique et 21 postes en orthopédie pédiatrique. Il existe six autres postes non ciblés, pouvant être occupés soit par un CCA à orientation viscérale, soit par un CCA à orientation orthopédique selon les cas.

Parmi les internes devant terminer leur internat au $1^{\text {er }}$ novembre 2011, quatre internes inscrits au DESC de chirurgie infantile n'auraient pas de poste de CCA.

\section{Discussion}

Avec $100 \%$ de réponses au questionnaire, notre objectif de recensement des internes et CCA en formation en chirurgie pédiatrique est atteint et permet 
Tableau III. Postes de chef de clinique-assistant (CCA) ou d'assistant hospitalo-universitaire (AHU) en chirurgie pédiatrique en mai 2010.

\begin{tabular}{|l|c|c|c|c|}
\hline Inter-région & $\begin{array}{c}\text { Uro-viscérale, } \\
\text { thoracique ou plastique }\end{array}$ & Orthopédie & Chirurgie pédiatrique & Total \\
\hline Nord & 4 & 2 & 1 & 7 \\
\hline Ouest & 5 & 4 & 0 & 9 \\
\hline Sud-Ouest & 2 & 2 & 1 & 5 \\
\hline Sud-Est & 3 & 3 & 1 & 7 \\
\hline $\begin{array}{l}\text { Rhône-Alpes } \\
\text { Auvergne }\end{array}$ & 4 & 2 & 1 & 7 \\
\hline Est & 2 & 2 & 2 & 6 \\
\hline Île-de-France & 8 & 6 & 0 & 14 \\
\hline Total & 28 & 21 & 6 & 55 \\
\hline
\end{tabular}

Dans la colonne « chirurgie pédiatrique», les postes peuvent être occupés par un chirurgien pédiatre quelle que soit son orientation, uro-viscérale, orthopédie ou autre.

une étude pertinente des tendances observées depuis la dernière étude démographique menée par le $\mathrm{CN}$ $\mathrm{HUCP}^{[2]}$. Le nombre d'internes qui s'engagent dans cette spécialité est en augmentation et annonciateur de difficultés à venir, tant à l'échelle individuelle pour trouver un débouché durant le post-internat et au-delà, qu'à l'échelle nationale (objectif de santé publique). Il est donc nécessaire de réguler la démographie des chirurgiens pédiatres en fonction des besoins de santé de la population et de mettre en adéquation les capacités de formation des CHU.

Après validation des ECN, il existe trois étapes majeures au cours de la formation chirurgicale pédiatrique : l'inscription en DESC de chirurgie infantile, l'obtention d'un poste de CCA ou d'AHU puis l'obtention d'un poste hospitalier (ou l'installation en secteur libéral) une fois la formation achevée.

\section{Inscriptions en DESC}

Le nombre d'internes formés chaque année n'est pas contrôlé de façon stricte et les recommandations nationales, non formulées par écrit, ne sont pas appliquées de la même manière selon les interrégions. Les internes souhaitant s'engager dans cette spécialité s'inscrivent en DESC auprès du coordonnateur inter-régional de leur spécialité, avec l'accord du doyen de la faculté. La CNIPI, conformément à la loi $n^{\circ}$ 2009-879 du 21 juillet 2009 portant réforme de l'hôpital et relative aux patients, à la santé et aux territoires, dite loi HPST, doit définir le nombre de postes ouverts aux nouveaux internes dans chaque spécialité $^{[3]}$. Le système des DESC pourrait également être réformé ${ }^{[4,5]}$. Une étude approfondie est nécessaire pour accorder le nombre de futurs chirurgiens pédiatres formés chaque année aux besoins de santé de la population et aux capacités de formation des centres hospitaliers universitaires. Ces données ne sont pas disponibles à ce jour.

\section{Le post-internat}

Les postes de CCA ou d'AHU existant actuellement ne suffisent pas à assurer une formation suffisante pour tous les internes inscrits en DESC. Quatre internes de chirurgie pédiatrique n'ont pas de poste disponible à la fin de leur internat, prévu en novembre 2011, comme c'est le cas dans d'autres spécialités médicales. En attendant un éventuel poste, les internes dans cette situation peuvent se consacrer à la recherche, à l'obtention de diplômes universitaires, ou encore poursuivre leur formation à l'étranger. Pour répondre à cette difficulté, 200 postes d'assistants spécialistes ont été créés pour l'année 2009 mais aucun poste n'a été attribué en chirurgie pédiatrique au moment de notre étude ${ }^{[6]}$. 
L'ouverture de 200 autres postes est prévue pour l'année 2010 mais la répartition de ces postes entre les différentes spécialités, y compris la médecine générale, n'est pas déterminée. La CNIPI doit exprimer des recommandations sur l'attribution de ces postes.

\section{L'exercice après le post-internat}

La chirurgie pédiatrique est une spécialité pratiquée par un nombre de chirurgiens beaucoup moins important que dans la majorité des autres spécialités chirurgicales. D'après l'atlas national de la démographie médicale de 2009, 230 chirurgiens exerçant la chirurgie infantile sont inscrits au Conseil national de l'Ordre des médecins, contre 3460 en chirurgie générale et 2607 en chirurgie orthopédique ${ }^{[7]} ; 70 \%$ exercent dans le secteur hospitalier public, $10 \%$ exercent en secteur libéral exclusif et $20 \%$ ont une activité mixte publique et libérale. La majorité des chirurgiens pédiatres exerce donc en secteur public. L'étude de la démographie en chirurgie pédiatrique menée par le CNHUCP en février 2007 a permis d'évaluer le nombre de chirurgiens pédiatres arrivant en fin d'activité dans les années à venir ${ }^{[2]}$. Ces données sont résumées dans la figure 1. Le nombre d'internes actuellement en formation étant supérieur aux nombre de départs à la retraite, il sera difficile pour eux de trouver un poste une fois leur formation terminée. Les postes hospitaliers sont adaptés aux besoins de santé de la population et aux contraintes budgétaires mais pas au nombre de spécialistes croissant formés chaque année ${ }^{[8,9]}$. Le $n u$ merus clausus augmente alors que le nombre de postes hospitaliers reste stable ${ }^{[10-12]}$. Les internes en chirurgie vont donc avoir des difficultés à trouver des débouchés à la fin de leur formation, ce qui est paradoxal dans un contexte de désaffection des disciplines chirurgicales par les étudiants en médecine $^{[13,14]}$.

Parmi les chirurgiens pédiatres en exercice actuellement, $34 \%$ sont des femmes et $66 \%$ des hommes. Au contraire, la proportion de femmes parmi les internes de chirurgie pédiatrique est de

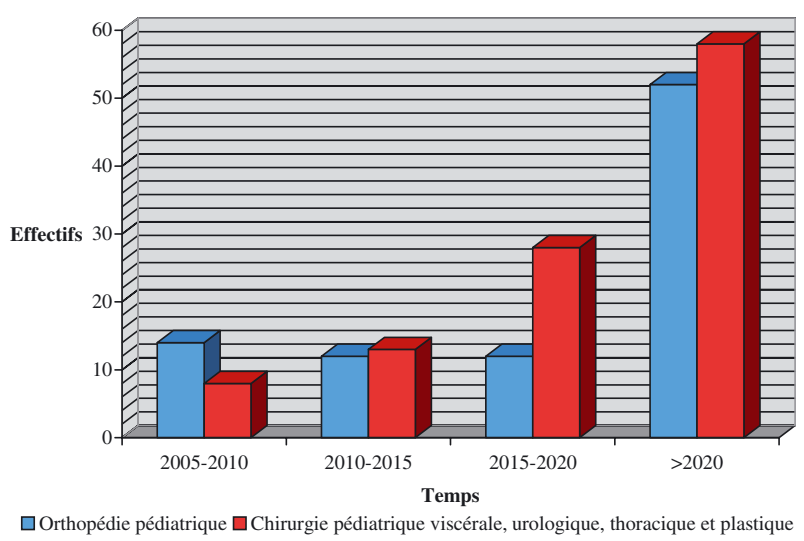

Fig. 1. Prévision par périodes de 5 ans du nombre de chirurgiens pédiatres arrivant en fin d'activité.

$66 \%$. Le ratio de femmes dans la spécialité va donc s'inverser et cette donnée est à prendre en compte car $80 \%$ des femmes chirurgiens pédiatres exercent exclusivement en secteur hospitalier contre $63 \%$ des hommes et plus souvent avec une activité à temps partiel. Le déficit de postes hospitaliers va donc progressivement s'aggraver.

\section{Le cas particulier de l'orthopédie pédiatrique}

Les chirurgiens qui exercent aujourd'hui l'orthopédie pédiatrique ont souvent suivi une formation d'orthopédie adulte. De même, treize internes actuellement inscrits en DESC d'orthopédie adulte prévoient de s'orienter ensuite vers une carrière en orthopédie pédiatrique. Il est donc possible d'exercer l'orthopédie pédiatrique après avoir validé soit le DESC de chirurgie orthopédique et traumatologique «adulte», soit le DESC de chirurgie infantile à orientation orthopédique et les habitudes en la matière sont très différentes selon les régions ${ }^{[15]}$. Cette situation rend l'analyse démographique difficile car on ne connaît pas le nombre exact d'internes d'orthopédie adulte souhaitant exercer en orthopédie pédiatrique, certains réservant leur réponse le plus tard possible afin de rester opportunistes, en fonction des postes qui s'offriront à eux. À ce propos, il faut rappeler qu'il existe une grande inégalité entre les spécialités chirurgicales adultes et pédiatriques puisque les chirurgiens pédiatres ont une activité 
exclusive chez l'enfant, alors que les chirurgiens d'adulte ont toujours la possibilité d'opérer des enfants, même s'ils n'ont jamais validé le moindre stage en chirurgie pédiatrique durant leur formation, et ce malgré les recommandations du schéma régional d'organisation sanitaire $3^{[16]}$, qui restent floues et souvent peu respectées ou mal organisées. Il faudrait envisager sans doute une activité chirurgicale pédiatrique minimale par département, qui pourrait s'effectuer en réseau avec un CHU, par exemple.

\section{Conclusion}

Cette étude précise la situation démographique des chirurgiens pédiatres en formation au $1^{\mathrm{er}}$ mai 2010 , à l'approche des réformes annoncées du troisième cycle des études médicales. La chirurgie pédiatrique est une spécialité très attractive pour les internes. Il est donc nécessaire d'adapter le nombre de futurs chirurgiens pédiatres aux capacités de formation de notre système de santé et aux besoins de santé de la population française.

La collaboration entre le Ministère de la Santé et des Sports, le Ministère de l'Éducation Nationale, la CNIPI, les collèges de spécialité et les associations étudiantes s'impose pour permettre une réforme constructive de l'internat et du post-internat.

\section{Contributions}

Aurélien Scalabre a conçu l'étude et contribué au recueil des données, à l'interprétation des résultats et à l'écriture du manuscrit; il est président de l'Association des chirurgiens pédiatres en formation (ACPF). Emeline Bourgeois, Matthieu Peycelon, Maguelonne Pons, respectivement viceprésident, trésorier et secrétaire de l'ACPF, ont participé au recueil des données. Sébastien Raux a participé au recueil des données. Rémi Kohler et François Varlet, respectivement président et secrétaire du Collège national de chirurgie pédiatrique, ont participé au recueil des données, à l'interprétation des résultats et à l'écriture du manuscrit. Les auteurs ne déclarent aucun conflit d'intérêt.

\section{Références}

1. Décret $\mathrm{n}^{\circ}$ 2009-272 du 9 mars 2009 relatif à la Commission nationale de l'internat et du postinternat NOR: SASH0902746D J.O. du 17 mars 2010. [On-line] Disponible sur : http:// www. legifrance.gouv. fr/affichTexte.do? cidTexte=JORFTEXTQ00020371725

2. Heloury Y, Démographie de la Spécialité : Rapport du Collège Hospitalier et Universitaire de Chirurgie Pédiatrique 2007 [On-line] Disponible sur : http://www.chirpediatric.fr/Les_textes/ Textes/demographie_CP.pdf

3. Loi $n^{\circ}$ 2009-879 du 21 juillet 2009 portant réforme de l'hôpital et relative aux patients, à la santé et aux territoires J.O. du 27 mars 2010. [On-line] Disponible sur : http://www . legifrance.gouv. fr/affichTexte.do; jsessionid=7629C7354363244707A2CDQBC8F 24389 . tpdjo11v_1?cidTexte=JORFTEXT000020879475\& categorieLien=id

4. Branchereau A, Benchimol D et le groupe de travail «Formation » pour le Conseil National de la Chirurgie Projet de réforme des DES et DESC des disciplines chirurgicales. 2006 [On-line] Disponible sur : http://web5 . unilim.fr/colneuro-test/IMG/ pdf_Projet_de_reforme_DES.pdf

5. Arrêté du 22 septembre 2004 fixant la liste et la réglementation des Diplômes d'Études Spécialisées en Médecine. J.O. du 6 octobre 2004. [On-line] Disponible sur : http:// www. legifrance.gouv. fr/affichTexte.do; jsessionid=FD1E8E6B5F5B98F0924AD95D50743638. tpdjo11v_1?cidTexte=JORFTEXT000000807238\& dateTexte=\&oldAction=rechJ0\& categorieLien=id

6. Circulaire DHOS/RH No. 2009-26 du 26 janvier 2009 relative au recrutement d'assistants spécialistes dans les CHU NOR : SJSH0930042CBO Santé - Protection sociale - Solidarités No. 2009/2 du 15 mars 2009. [Online] Disponible sur : http://adiph.asso.fr/TO/ circ260109-assistantsCHU.pdf

7. Le Breton-Lerouvillois G, Legmann M, Kahn-Bensaude I, Romestaing P. Atlas de la démographie médicale en France. Situation au $1^{\text {er }}$ janvier 2009. Conseil National de l'Ordre des Médecins. 2009 [On-line] Disponible sur : http: //www . conseil-national . medecin. fr/ sites/default/files/atlas2009_0.pdf

8. Commission Démographie médicale. Rapport présenté par Berland Y. 2006. [On-line] Disponible sur: http:// www . securite-sociale. fr/communications/ rapports/2006/berland/rapport_berland.pdf 
9. Langlois J. La démographie médicale de 2003 à 2025. Difficultés présentes et à venir. Bull Acad Natl Méd 2004;188:675-93.

10. Doan BD, Levy D, Pavot J. Projection démographique de la profession médicale en France (2000-2050). Quel numerus clausus pour quel avenir ? Cahiers de Sociologie Démographie Médicale 2004;44: 101-48.

11. Roussel F. Épreuves Classantes Nationales, une inadéquation majeure entre le nombre des postes et celui des candidats. Presse Med 2006;35:437-8.

12. Roupret M, Hupertan V, Chartier-Kastler E. The choice of a medical career in a population of 600 second-cycle French médical students preparing the national-ranking exam. Presse Med 2005;34:78690.

13. Maggiori L, Brouquet A, Zeitoun JD, Roupret M, Lefevre $\mathrm{JH}$ The future of gastrointestinal surgery in France: national survey of 929 medical students and results of professional choices after the 2008 national ranking exam. J Chir 2009;146:168-74.
14. Roupret M, Pouliquen Y. Should French surgery been helped? Presse Med 2004;33:1411-3.

15. Caton J, Duburcq A. Basis for an evaluation of needs of orthopaedic surgeons in France. Rev Chir Orthop Reparatrice Appar Mot 2006;92:818-26.

16. Circulaire $\mathrm{N}^{\circ} 517 / \mathrm{DHOS} / \mathrm{O} 1 / \mathrm{DGS} / \mathrm{DGAS}$ du 28 octobre 2004 relative à l'élaboration des SROS de l'enfant et de l'adolescent NOR : SANH0430649C. 2004 [On-line] Disponible sur : http://www.gfrup.com/texte_ officiel/snpeh_sros_pediatrie_circulaire. $\operatorname{pdf}$

Correspondance et offprints : A. Scalabre, Service de pédiatique, Hôpital Femme-Mère-Enfant, 59 boulevard Pinel, 69500 Bron, France

Mailto : aurelien.scalabre@chu_lyon.fr 